\title{
Structural analysis by stochastic differential scanning calorimetry
}

\author{
Alex Sherman ${ }^{a}$, Casey Smith ${ }^{b}$, Andreas Geiger ${ }^{c}$, Paul Stroud ${ }^{d}$, Jeremy Hinds ${ }^{\mathrm{e}}$, and Garth \\ Simpson ${ }^{f^{*}}$ \\ aPurdue University, 560 Oval Drive, West Lafayette, Indiana 47907, USA, sherma10@purdue.edu \\ bPurdue University, 560 Oval Drive, West Lafayette, Indiana 47907, USA, smit2698@purdue.edu \\ cPurdue University, 560 Oval Drive, West Lafayette, Indiana 47907, USA, geiger15@purdue.edu \\ dEli Lilly and Company, Indianapolis, Indiana, USA, stroud paul andrew@lilly.com \\ eEli Lilly and Company, Indianapolis, Indiana, USA, hinds jeremy@lilly.com \\ f*Purdue University, 560 Oval Drive, West Lafayette, Indiana 47907, USA, gsimpson@purdue.edu
}

Integration of second harmonic generation (SHG) imaging with differential scanning calorimetry (DSC) enables a thermal and physical analysis of single-particle stochastic phase transformations in organic crystals, which collectively contribute to the ensemble-averaged thermal transients interrogated by DSC. The SHG-activity of a crystal is highly sensitive to the specific molecular packing arrangement within a noncentrosymmetric lattice, providing access to structural information otherwise unavailable by conventional imaging approaches. ${ }^{1,2}$ Consequently, lattice transformations associated with dehydration/desolvation events were observed by SHG imaging and correlated to phase transformations observed in the DSC measurements.

Following the studies of a model system, stochastic differential scanning calorimetry (SDSC) was performed to interrogate samples of crystalline trehalose dihydrate which exhibit complex phasetransition behaviors. ${ }^{3}$ In general, the structural analysis of trehalose dihydrate during heating is complicated by the existence of varying polymorphs that form under different conditions, leading to an ongoing debate on how the polymorphs of trehalose are connected to each other during various thermal/phase transformations. ${ }^{4}$ Since significant structural differences can arise due to polymorphic transition, trehalose dihydrate was analyzed by SDSC using an integrated DSC-SHG microscope to probe crystal form throughout its phase transitions.

\section{$\underline{\text { References }}$}

[1] Chowdhury, A. U. et al. Kinetic trapping of metastable amino acid polymorphs. Journal of the American Chemical Society 136, 2404-2412 (2014).

[2] Kestur, U. S. et al. Nonlinear optical imaging for sensitive detection of crystals in bulk amorphous powders. Journal of pharmaceutical sciences 101, 4201-4213 (2012).

[3] Sussich, F., Urbani, R., Princivalle, F. \& Cesaro, A. Polymorphic amorphous and crystalline forms of trehalose. J. Am. Chem. Soc. 120, 7893-7899 (1998).

[4] Singh, S. K. in Challenges in Protein Product Development 63-95 (Springer, 2018). 